\title{
Fuzzy fixed point theorems on the complete fuzzy spaces under supremum metric
}

\author{
Suthep Suantai ${ }^{1}$, Narin Petrot ${ }^{2,3^{*}}$ and Warut Saksirikun ${ }^{2}$
}

\author{
"Correspondence: narinp@nu.ac.th \\ 2Department of Mathematics, \\ Faculty of Science, Naresuan \\ University, Phitsanulok, 65000, \\ Thailand \\ ${ }^{3}$ Research Center for Academic \\ Excellence in Mathematics, Faculty \\ of Science, Naresuan University, \\ Phitsanulok, 65000, Thailand \\ Full list of author information is \\ available at the end of the article
}

\begin{abstract}
By using the concept of a class of functions, the $\mathcal{R}$-functions, we provide some fuzzy fixed point theorems on a space of fuzzy sets equipped with the supremum metric. By presenting a technique of constructing a sequence of successive approximations, we obtain some interesting results that improve many existing results. The related cases are also shown and discussed.
\end{abstract}

MSC: $47 \mathrm{H} 10 ; 47 \mathrm{~S} 40 ; 54 \mathrm{H} 25$

Keywords: fuzzy set; supremum metric; fuzzy mapping; fuzzy fixed point; $\mathcal{R}$-functions

\section{Introduction}

By using a natural generalization of the concept of a set, the fuzzy set, which was introduced initially by Zadeh [1], considering mathematical programming problems which are expressed as optimizing some goal function given certain constraints, this be relaxed by means of a subjective gradation. In 1981, Heilpern [2] used the concept of fuzzy sets and introduced a class of fuzzy mappings, which is a generalization of the set-valued mapping, and proved a fixed point theorem for fuzzy contraction mappings in metric linear spaces. It is worth noting that the result announced by Heilpern [2] is a fuzzy extension of the Banach contraction principle. Subsequently, several other authors have studied the existence of fixed points of fuzzy mappings; for example, Estruch and Vidal [3] proved a fixed point theorem for fuzzy contraction mappings over a complete metric space, which is a generalization of the given Heilpern fixed point theorem, and Sedghi et al. [4] gave an extended version of the Estruch and Vidal [3] theorem (for more examples, see [5-14]).

Although many kinds of fixed point theorems for fuzzy contraction mappings in complete metric spaces have been studied extensively in recent years, we have to point out that one has given most attention to the class of fuzzy sets with nonempty compact $\alpha$-cut sets in the metric space $X$, but little attention to the class of fuzzy sets with nonempty bounded or closed, or even bounded closed, $\alpha$-cut sets. However, it is well known that all compact sets are bounded closed sets in a general metric space and the converse is not always true.

In 2008, Qui and Shu [12] established the completeness of $\mathcal{C B}(X)$ with respect to the completeness of the metric space $X$, where $\mathcal{C B}(X)$ denotes the class of fuzzy sets with

(c) 2015 Suantai et al. This article is distributed under the terms of the Creative Commons Attribution 4.0 International License (http://creativecommons.org/licenses/by/4.0/), which permits unrestricted use, distribution, and reproduction in any medium, provided you give appropriate credit to the original author(s) and the source, provide a link to the Creative Commons license, and indicate if changes were made. 
nonempty bounded closed $\alpha$-cut sets equipped with the generalized Hausdorff metric $d_{\infty}$, which takes the supremum on the Hausdorff distances between the corresponding $\alpha$-cut sets. Also they proved the following common fixed point theorem for a family of fuzzy mappings.

Theorem 1.1 [12] Let $(X, d)$ be a complete metric space and let $\left\{F_{i}\right\}$ be a sequence of selfmappings of $\mathcal{C B}(X)$. If there exists a constant $q \in(0,1)$ such that for each $\mu_{1}, \mu_{2} \in \mathcal{C B}(X)$, and for arbitrary positive integers $i$ and $j, i \neq j$,

$$
d_{\infty}\left(F_{i}\left(\mu_{1}\right), F_{j}\left(\mu_{2}\right)\right) \leq q M_{(i, j)}\left(\mu_{1}, \mu_{2}\right),
$$

where

$$
\begin{aligned}
M_{(i, j)}\left(\mu_{1}, \mu_{2}\right)= & \max \left\{d_{\infty}\left(\mu_{1}, \mu_{2}\right), \rho_{\infty}\left(\mu_{1}, F_{i}\left(\mu_{1}\right)\right), \rho_{\infty}\left(\mu_{2}, F_{j}\left(\mu_{2}\right)\right),\right. \\
& \left.\frac{\rho_{\infty}\left(\mu_{2}, F_{i}\left(\mu_{1}\right)\right)+\rho_{\infty}\left(\mu_{1}, F_{j}\left(\mu_{2}\right)\right)}{2}\right\} .
\end{aligned}
$$

Then there exists a $\mu_{*} \in \mathcal{C B}(X)$ such that $\mu_{*} \subseteq F_{i}\left(\mu_{*}\right)$ for all $i \in \mathbb{N}$.

Later, by using the concept of $d_{\infty}$ metric, Qiu et al. [15] proved the following common fixed point theorem, but under the assumption of a compact cut set $\mathcal{C}(X)$ instead of a closed bounded cut set $\mathcal{C B}(X)$.

Theorem 1.2 [15] Let $(X, d)$ be a compact metric space and let $\left\{F_{i}\right\}_{i=1}^{\infty}$ be a sequence of self-mappings of $\mathcal{C}(X)$. Let $\Phi:[0, \infty) \rightarrow[0, \infty)$ be a non-decreasing function satisfying the following condition: $\Phi$ is continuous from the right and

$$
\sum_{n=1}^{\infty} \Phi^{n}(t)<\infty, \quad \text { for all } t>0,
$$

where $\Phi^{n}$ denotes the nth iterative function of $\Phi$. Suppose that for arbitrary positive integers $i$ and $j, i \neq j$,

$$
d_{\infty}\left(F_{i}\left(\mu_{1}\right), F_{j}\left(\mu_{2}\right)\right) \leq \Phi\left(M_{(i, j)}\left(\mu_{1}, \mu_{2}\right)\right),
$$

where $M_{(i, j)}\left(\mu_{1}, \mu_{2}\right)$ is defined as in Theorem 1.1. Then there exists a $\mu_{*} \in \mathcal{C}(X)$ such that $\mu_{*} \subseteq F_{i}\left(\mu_{*}\right)$ for all $i \in \mathbb{N}$.

Notice that Theorem 1.2 can be used to apply to a larger class of mappings than that of Theorem 1.1. However, after careful consideration, one may see that Theorem 1.2 is relevant when the considered space is a compact metric space instead of a complete metric space, which has been considered in Theorem 1.1.

Based on the above remarks, here we will present an extension of Theorem 1.1, but in a complete metric space setting. In fact, we will use the concept of a class of functions, so-called $\mathcal{R}$-functions, to show some fixed point theorems for self-mappings of $\mathcal{C B}(X)$ with the supremum metric for fuzzy sets. Of course, our results improve and extend those results which have been presented in [12] and [15]. 


\section{Preliminaries}

In this section, we will provide some important basic concepts and useful results. Let $(X, d)$ be a metric space, and let $C B(X)$ be the set of all nonempty bounded closed subsets of $X$. Recall that the Hausdorff metric is a function $H$ on $C B(X)$ defined by

$$
\begin{aligned}
H(A, B) & =\max \left\{\sup _{x \in B} d(x, A), \sup _{x \in A} d(x, B)\right\} \\
& :=\max \{\rho(B, A), \rho(A, B)\}, \quad \text { for all } A, B \in C B(X),
\end{aligned}
$$

where $\rho(A, B)=\sup _{x \in A} d(x, B)$ is the Hausdorff separation of $A$ from $B$.

\subsection{Fuzzy sets and fuzzy mappings}

Let $I=[0,1]$. A fuzzy set $\mu$ of a metric space $X$ is defined by its membership function $\mu(x)$, which is a mapping from $X$ into $I$. We denote by $\mathfrak{F}(X)$ the set of all fuzzy mappings on $X$, that is, $\mathfrak{F}(X):=\{\mu \mid \mu: X \rightarrow[0,1]\}$. For any $\alpha \in(0,1]$, the $\alpha$-cut of the fuzzy set $\mu$ is defined by

$$
[\mu]_{\alpha}=\{x \in X: \mu(x) \geq \alpha\}
$$

where $\alpha \in(0,1]$, and we separately specify the support $[\mu]_{0}$ of $\mu$ to be the closure of the union of $[\mu]_{\alpha}$ for $0<\alpha \leq 1$. We denote by $\mathcal{C B}(X)$ the totality of fuzzy sets $\mu: X \rightarrow I$ for which, for each $\alpha \in I$, the $\alpha$-cut of $\mu$ is a nonempty closed bounded subset of $X$.

Let $\mu_{1}, \mu_{2} \in \mathfrak{F}(X)$. Then $\mu_{1}$ is said to be included in $\mu_{2}$, denoted by $\mu_{1} \subseteq \mu_{2}$, if and only if $\mu_{1}(x) \leq \mu_{2}(x)$ for each $x \in X$. Thus we have $\mu_{1} \subseteq \mu_{2}$ if and only if $\left[\mu_{1}\right]^{\alpha} \subseteq\left[\mu_{2}\right]^{\alpha}$ for all $\alpha \in I$. Let $X, Y$ be any underling sets and $\mathfrak{U}, \mathfrak{V}$ are subsets of $\mathfrak{F}(X)$ and $\mathfrak{F}(Y)$, respectively. A mapping $F: \mathfrak{U} \rightarrow \mathfrak{V}$ is said to be a fuzzy mapping, i.e., $F(\mu) \in \mathfrak{V}$ for each $\mu \in \mathfrak{U}$. An element $\mu_{*} \in \mathfrak{U}$ is said to be a fixed point of a fuzzy self-mapping $F$ on $\mathfrak{U}$ if and only if $\mu_{*} \subseteq F\left(\mu_{*}\right)$.

The $d_{\infty}$-metric (called supremum or generalized Hausdorff metric) is a metric on $\mathcal{C B}(X)$ which is defined as follows:

$$
\begin{aligned}
d_{\infty}\left(\mu_{1}, \mu_{2}\right) & =\sup _{0 \leq \alpha \leq 1} H\left(\left[\mu_{1}\right]^{\alpha},\left[\mu_{2}\right]^{\alpha}\right) \\
& =\max \left\{\rho_{\infty}\left(\mu_{1}, \mu_{2}\right), \rho_{\infty}\left(\mu_{2}, \mu_{1}\right)\right\},
\end{aligned}
$$

where $\mu_{1}, \mu_{2} \in \mathcal{C B}(X)$, and

$$
\rho_{\infty}\left(\mu_{1}, \mu_{2}\right)=\sup _{0 \leq \alpha \leq 1} \rho\left(\left[\mu_{1}\right]^{\alpha},\left[\mu_{2}\right]^{\alpha}\right)
$$

is the Hausdorff separation of $\mu_{1}$ from $\mu_{2}$. Notice that the supremum in (2.1) may be not attained, and so it cannot be replaced by a maximum. To clarify this, we include the following example, which can be found in [16].

Example 2.1 Let $X$ be a set of real numbers and $\mu, v \in \mathfrak{F}(X)$ be fuzzy subsets of $X$ such that the corresponding level sets are

$$
[\mu]^{\alpha}=[v]^{\alpha}=[0,1] \text { for } 0 \leq \alpha \leq \frac{1}{2},
$$


and

$$
[\mu]^{\alpha}=\{0\}, \quad[v]^{\alpha}=[0,2(1-\alpha)] \text { for } \frac{1}{2} \leq \alpha \leq 1 .
$$

It follows that

$$
H\left([\mu]^{\alpha},[v]^{\alpha}\right)= \begin{cases}0 & \text { for } 0 \leq \alpha \leq \frac{1}{2} \\ 2(1-\alpha) & \text { for } \frac{1}{2} \leq \alpha \leq 1\end{cases}
$$

Then $d_{\infty}(\mu, v)=\sup _{0 \leq \alpha \leq 1} H\left([\mu]^{\alpha},[v]^{\alpha}\right)=1$, but this is not attained.

Note that if $\left\{\mu_{n}\right\}$ be a sequence in $\mathcal{C B}(X)$, then it follows from the definition of $d_{\infty}$ that $\left\{\mu_{n}\right\}$ converges with respect to the $d_{\infty}$-metric if and only if $\left[\mu_{n}\right]^{\alpha}$ converges uniformly in $\alpha \in I$ with respect to the Hausdorff metric. Further, we know that the metric space $\left(\mathcal{C B}(X), d_{\infty}\right)$ is complete provided $(X, d)$ is complete (see [12]). Here, we collect some useful properties of the $d_{\infty}$-metric, which will be used in order to obtain our results.

Lemma 2.2 [12] Let $\mu_{1}, \mu_{2}, \mu_{3} \in \mathcal{C B}(X)$. The following items are true:

(i) $\rho_{\infty}\left(\mu_{1}, \mu_{2}\right)=0$ if and only if $\mu_{1} \subseteq \mu_{2}$,

(ii) if $\mu_{1} \subseteq \mu_{2}$, then $\rho_{\infty}\left(\mu_{1}, \mu_{3}\right) \leq d_{\infty}\left(\mu_{2}, \mu_{3}\right)$,

(iii) $\rho_{\infty}\left(\mu_{1}, \mu_{3}\right) \leq d_{\infty}\left(\mu_{1}, \mu_{2}\right)+\rho_{\infty}\left(\mu_{2}, \mu_{3}\right)$.

Theorem 2.3 [12] Let $(X, d)$ be a metric space and $\mu_{1}, \mu_{2} \in \mathcal{C B}(X)$. Then for any $\beta>1$ and any $\mu_{3} \in \mathcal{C B}(X)$ satisfying $\mu_{3} \subseteq \mu_{1}$, there exists a $\mu_{4} \in \mathcal{C B}(X)$ such that $\mu_{4} \subseteq \mu_{2}$ and $d_{\infty}\left(\mu_{3}, \mu_{4}\right) \leq \beta d_{\infty}\left(\mu_{1}, \mu_{2}\right)$.

\section{$2.2 \mathcal{R}$-Functions}

In this subsection, we will recall an important tool related to our considered class of mappings. A function $\varphi:[0, \infty) \rightarrow[0,1)$ is said to be an $\mathcal{R}$-function if

$$
\limsup _{s \rightarrow t^{+}} \varphi(s)<1 \quad \text { for all } t \in[0, \infty)
$$

Note that if $\varphi:[0, \infty) \rightarrow[0,1)$ is a non-decreasing function or a non-increasing function, then $\varphi$ is an $\mathcal{R}$-function. This means the set of $\mathcal{R}$-functions is a rich class. In [17], Du proved some of the following characterizations for the class of $\mathcal{R}$-functions.

Theorem 2.4 [17] Let $\varphi:[0, \infty) \rightarrow[0,1)$ be a function. Then the following statements are equivalent.

(a) $\varphi$ is an $\mathcal{R}$-function.

(b) For any nonincreasing sequence $\left\{x_{n}\right\}_{n \in \mathbb{N}}$ in $[0, \infty)$, we have $0 \leq \sup _{n \in \mathbb{N}} \varphi\left(x_{n}\right)<1$.

\section{Fixed point theorems for fuzzy mappings induced by $\mathcal{R}$-functions}

Now, we are in a position to present our main results.

Theorem 3.1 Let $(X, d)$ be a complete metric space and let $\left\{F_{i}\right\}_{i=1}^{\infty}$ be a sequence of fuzzy self-mappings of $\mathcal{C B}(X)$. Assume that there exists an $\mathcal{R}$-function $\varphi:[0, \infty) \rightarrow[0,1)$ such 
that for each $\mu_{1}, \mu_{2} \in \mathcal{C B}(X)$, and for arbitrary positive integers $i$ and $j, i \neq j$,

$$
d_{\infty}\left(F_{i}\left(\mu_{1}\right), F_{j}\left(\mu_{2}\right)\right) \leq \varphi\left(d_{\infty}\left(\mu_{1}, \mu_{2}\right)\right) M_{(i, j)}\left(\mu_{1}, \mu_{2}\right)
$$

where $M_{(i, j)}\left(\mu_{1}, \mu_{2}\right)$ is defined as in Theorem 1.1. Then there exists a $\mu_{*} \in \mathcal{C B}(X)$ such that $\mu_{*} \subseteq F_{i}\left(\mu_{*}\right)$ for all positive integers $i$.

Proof Let us define a function $k:[0, \infty) \rightarrow[0,1)$ by

$$
k(t)=\frac{1+\varphi(t)}{2}, \quad \text { for all } t \in[0, \infty)
$$

Note that we have $0 \leq \varphi(t)<k(t)<1$ for all $t \in[0, \infty)$.

We will start by picking a fuzzy set $\mu_{0} \in \mathcal{C B}(X)$. We subsequently choose $\mu_{1} \subseteq F_{1}\left(\mu_{0}\right)$ and a positive real number $\varepsilon_{0}$ such that $\varepsilon_{0} \in\left(\frac{1-k\left(d_{\infty}\left(\mu_{0}, \mu_{1}\right)\right)}{2}, 1-k\left(d_{\infty}\left(\mu_{0}, \mu_{1}\right)\right)\right)$. Next, by using this $\varepsilon_{0}$, we can find a positive real number $\beta_{0}$ such that $\beta_{0} \in\left(1, \frac{1-\varepsilon_{0}}{k\left(d_{\infty}\left(\mu_{0}, \mu_{1}\right)\right)}\right)$. Now, by Theorem 2.3, there exists $\mu_{2} \in \mathcal{C B}(X)$ such that $\mu_{2} \subseteq F_{2}\left(\mu_{1}\right)$ and

$$
d_{\infty}\left(\mu_{1}, \mu_{2}\right) \leq \beta_{0} d_{\infty}\left(F_{1}\left(\mu_{0}\right), F_{2}\left(\mu_{1}\right)\right) .
$$

Next, let us to choose a positive real number $\varepsilon_{1}$ such that $\varepsilon_{1} \in\left(\frac{1-k\left(d_{\infty}\left(\mu_{1}, \mu_{2}\right)\right)}{2}, 1-\right.$ $\left.k\left(d_{\infty}\left(\mu_{1}, \mu_{2}\right)\right)\right)$, and then pick a positive real number $\beta_{1}$ such that $\beta_{1} \in\left(1, \frac{1-\varepsilon_{1}}{k\left(d_{\infty}\left(\mu_{1}, \mu_{2}\right)\right)}\right)$. Similarly to the above, by Theorem 2.3 , we can find $\mu_{3} \in \mathcal{C B}(X)$ such that $\mu_{3} \subseteq F_{3}\left(\mu_{2}\right)$ and

$$
d_{\infty}\left(\mu_{2}, \mu_{3}\right) \leq \beta_{1} d_{\infty}\left(F_{2}\left(\mu_{1}\right), F_{3}\left(\mu_{2}\right)\right) .
$$

By continuing this process, we obtain two sequences of positive real numbers $\left\{\varepsilon_{n}\right\},\left\{\beta_{n}\right\}$ and a sequence $\left\{\mu_{n}\right\}$ in $\mathcal{C B}(X)$ such that

$$
\left\{\begin{array}{l}
\mu_{n+1} \subseteq F_{n+1}\left(\mu_{n}\right) \\
d_{\infty}\left(\mu_{n+1}, \mu_{n+2}\right) \leq \beta_{n} d_{\infty}\left(F_{n+1}\left(\mu_{n}\right), F_{n+2}\left(\mu_{n+1}\right)\right) \\
\varepsilon_{n} \in\left(\frac{1-k\left(d_{\infty}\left(\mu_{n}, \mu_{n+1}\right)\right)}{2}, 1-k\left(d_{\infty}\left(\mu_{n}, \mu_{n+1}\right)\right)\right. \\
\beta_{n} \in\left(1, \frac{1-\varepsilon_{n}}{k\left(d_{\infty}\left(\mu_{n}, \mu_{n+1}\right)\right)}\right)
\end{array}\right.
$$

for each $n \in \mathbb{N}$.

In order to complete the proof, we will divide it into three steps.

Step 1 . We show that $\sup _{n \in \mathbb{N}} \beta_{n} k\left(d_{\infty}\left(\mu_{n}, \mu_{n+1}\right)\right)<1$.

Note that, by Lemma 2.2(iii), we have

$$
\rho_{\infty}\left(\mu_{n-1}, F_{n}\left(\mu_{n-1}\right)\right) \leq d_{\infty}\left(\mu_{n-1}, \mu_{n}\right)+\rho_{\infty}\left(\mu_{n}, F_{n}\left(\mu_{n-1}\right)\right)
$$

for each $n \in \mathbb{N}$. Subsequently, since $\mu_{n} \subseteq F_{n}\left(\mu_{n-1}\right)$, in view of Lemma 2.2(i) we obtain

$$
\rho_{\infty}\left(\mu_{n-1}, F_{n}\left(\mu_{n-1}\right)\right) \leq d_{\infty}\left(\mu_{n-1}, \mu_{n}\right)
$$

for each $n \in \mathbb{N}$. 
Using this fact, we now derive

$$
\begin{aligned}
& M_{(n+1, n+2)}\left(\mu_{n}, \mu_{n+1}\right) \\
&=\max \left\{d_{\infty}\left(\mu_{n}, \mu_{n+1}\right), \rho_{\infty}\left(\mu_{n}, F_{n+1}\left(\mu_{n}\right)\right), \rho_{\infty}\left(\mu_{n+1}, F_{n+2}\left(\mu_{n+1}\right)\right),\right. \\
&\left.1 / 2\left[\rho_{\infty}\left(\mu_{n+1}, F_{n+1}\left(\mu_{n}\right)\right)+\rho_{\infty}\left(\mu_{n}, F_{n+2}\left(\mu_{n+1}\right)\right)\right]\right\} \\
& \leq \max \left\{d_{\infty}\left(\mu_{n}, \mu_{n+1}\right), d_{\infty}\left(\mu_{n}, \mu_{n+1}\right), d_{\infty}\left(\mu_{n+1}, \mu_{n+2}\right), 1 / 2\left[d_{\infty}\left(\mu_{n}, \mu_{n+2}\right)\right.\right. \\
&\left.\left.+\rho_{\infty}\left(\mu_{n+2}, F_{n+2}\left(\mu_{n+1}\right)\right)\right]\right\} \\
& \leq \max \left\{d_{\infty}\left(\mu_{n}, \mu_{n+1}\right), d_{\infty}\left(\mu_{n+1}, \mu_{n+2}\right), 1 / 2\left[d_{\infty}\left(\mu_{n}, \mu_{n+1}\right)+d_{\infty}\left(\mu_{n+1}, \mu_{n+2}\right)\right]\right\} \\
&= \max \left\{d_{\infty}\left(\mu_{n}, \mu_{n+1}\right), d_{\infty}\left(\mu_{n+1}, \mu_{n+2}\right)\right\} .
\end{aligned}
$$

Using this one, in view of the inequalities (3.1) and (3.2), we obtain

$$
d_{\infty}\left(\mu_{n+1}, \mu_{n+2}\right)<\beta_{n} k\left(d_{\infty}\left(\mu_{n}, \mu_{n+1}\right)\right) \max \left\{d_{\infty}\left(\mu_{n}, \mu_{n+1}\right), d_{\infty}\left(\mu_{n+1}, \mu_{n+2}\right)\right\} .
$$

Then, by inequality (3.3), we must have

$$
\max \left\{d_{\infty}\left(\mu_{n}, \mu_{n+1}\right), d_{\infty}\left(\mu_{n+1}, \mu_{n+2}\right)\right\}=d_{\infty}\left(\mu_{n}, \mu_{n+1}\right)
$$

Indeed, if (3.4) is not true, we would have

$$
d_{\infty}\left(\mu_{n+1}, \mu_{n+2}\right)<\beta_{n} k\left(d_{\infty}\left(\mu_{n}, \mu_{n+1}\right)\right) d_{\infty}\left(\mu_{n+1}, \mu_{n+2}\right)
$$

by inequality (3.3). This leads to a contradiction, since $0<\beta_{n} k\left(d_{\infty}\left(\mu_{n}, \mu_{n+1}\right)\right)<1-\varepsilon_{n}<1$ for all $n \in \mathbb{N}$. This shows that (3.4) holds. Subsequently, since $\beta_{n} k\left(d_{\infty}\left(\mu_{n}, \mu_{n+1}\right)\right) \in(0,1)$, we have

$$
\begin{aligned}
d_{\infty}\left(\mu_{n+1}, \mu_{n+2}\right) & <\beta_{n} k\left(d_{\infty}\left(\mu_{n}, \mu_{n+1}\right)\right) d_{\infty}\left(\mu_{n}, \mu_{n+1}\right) \\
& <d_{\infty}\left(\mu_{n}, \mu_{n+1}\right) .
\end{aligned}
$$

This means the sequence $\left\{d_{\infty}\left(\mu_{n}, \mu_{n+1}\right)\right\}$ is strictly decreasing in $[0, \infty)$. Thus, by applying Theorem 2.4, we know that

$$
0 \leq \sup _{n \in \mathbb{N}} \varphi\left(d_{\infty}\left(\mu_{n}, \mu_{n+1}\right)\right)<1
$$

This implies

$$
\sup _{n \in \mathbb{N}} k\left(d_{\infty}\left(\mu_{n}, \mu_{n+1}\right)\right)=\frac{1}{2}\left[1+\sup _{n \in \mathbb{N}} \varphi\left(d_{\infty}\left(\mu_{n}, \mu_{n+1}\right)\right)\right]<1 .
$$

On the other hand, we observe that

$$
\begin{aligned}
\beta_{n} k\left(d_{\infty}\left(\mu_{n}, \mu_{n+1}\right)\right) & <1-\varepsilon_{n} \\
& <1-\left(\frac{1-k\left(d_{\infty}\left(\mu_{n}, \mu_{n+1}\right)\right)}{2}\right) \\
& =\frac{1+k\left(d_{\infty}\left(\mu_{n}, \mu_{n+1}\right)\right)}{2} .
\end{aligned}
$$


Using this together with (3.6), we can conclude that

$$
\sup _{n \in \mathbb{N}} \beta_{n} k\left(d_{\infty}\left(\mu_{n}, \mu_{n+1}\right)\right)<1
$$

as required.

Step 2 . We show that $\left\{\mu_{n}\right\}$ is a Cauchy sequence in $\mathcal{C B}(X)$.

According to step 1 , let us put $c:=\sup _{n \in \mathbb{N}} \beta_{n} k\left(d_{\infty}\left(\mu_{n}, \mu_{n+1}\right)\right) \in(0,1)$. Then, by (3.5), we have

$$
\begin{aligned}
d_{\infty}\left(\mu_{n+1}, \mu_{n+2}\right) & <\beta_{n} k\left(d_{\infty}\left(\mu_{n}, \mu_{n+1}\right)\right) d_{\infty}\left(\mu_{n}, \mu_{n+1}\right) \\
& \leq c d_{\infty}\left(\mu_{n}, \mu_{n+1}\right)
\end{aligned}
$$

for each $n \in \mathbb{N}$. By using this relation, we deduce that

$$
d_{\infty}\left(\mu_{n+1}, \mu_{n+2}\right)<c d_{\infty}\left(\mu_{n}, \mu_{n+1}\right)<\cdots<c^{n+1} d_{\infty}\left(\mu_{0}, \mu_{1}\right),
$$

for each $n \in \mathbb{N}$.

So, for arbitrary positive integers $m$ and $k$, we see that

$$
\begin{aligned}
d_{\infty}\left(\mu_{k}, \mu_{k+m}\right) & \leq \sum_{i=k}^{k+m-1} d_{\infty}\left(\mu_{i}, \mu_{i+1}\right) \\
& <\sum_{i=k}^{k+m-1} c^{i} d_{\infty}\left(\mu_{0}, \mu_{1}\right) \\
& \leq \frac{c^{k}}{1-c} d_{\infty}\left(\mu_{0}, \mu_{1}\right)
\end{aligned}
$$

Since $c \in(0,1)$, we can conclude that $\left\{\mu_{n}\right\}$ is a Cauchy sequence in $\mathcal{C B}(X)$, as required.

Step 3. We show that there is $\mu_{*} \in \mathcal{C B}(X)$ such that $\mu_{*} \subset F_{i}\left(\mu_{*}\right)$, for all $i \in \mathbb{N}$.

Since $\left\{\mu_{n}\right\}$ is a Cauchy sequence in a complete metric space $\left(\mathcal{C B}(X), d_{\infty}\right)$, there is $\mu_{*} \in$ $\mathcal{C B}(X)$ such that $\mu_{n} \rightarrow \mu_{*}$ as $n \rightarrow \infty$. We now show that $\mu_{*} \subseteq F_{i}\left(\mu_{*}\right)$ for all $i \in \mathbb{N}$.

Let $i \in \mathbb{N}$ be arbitrary. Firstly, by Lemma 2.2(iii) and (iv), let us notice that

$$
\begin{aligned}
\rho_{\infty}\left(\mu_{*}, F_{i}\left(\mu_{*}\right)\right) & \leq d_{\infty}\left(\mu_{*}, \mu_{j}\right)+\rho_{\infty}\left(\mu_{j}, F_{i}\left(\mu_{*}\right)\right) \\
& \leq d_{\infty}\left(\mu_{*}, \mu_{j}\right)+d_{\infty}\left(F_{j}\left(\mu_{j-1}\right), F_{i}\left(\mu_{*}\right)\right),
\end{aligned}
$$

since $\mu_{j} \subset F_{j}\left(\mu_{j-1}\right)$ for arbitrary natural numbers $j$ such that $i \neq j$.

Subsequently, by using (3.7) and Lemma 2.2(iii), we derive

$$
\begin{aligned}
d_{\infty}\left(F_{j}\left(\mu_{j-1}\right), F_{i}\left(\mu_{*}\right)\right) \leq & \varphi\left(d_{\infty}\left(\mu_{j-1}, \mu_{*}\right)\right) \max \left\{d_{\infty}\left(\mu_{j-1}, \mu_{*}\right), \rho_{\infty}\left(\mu_{j-1}, F_{j}\left(\mu_{j-1}\right)\right),\right. \\
& \left.\rho_{\infty}\left(\mu_{*}, F_{i}\left(\mu_{*}\right)\right), 1 / 2\left[\rho_{\infty}\left(\mu_{*}, F_{j}\left(\mu_{j-1}\right)\right)+\rho_{\infty}\left(\mu_{j-1}, F_{i}\left(\mu_{*}\right)\right)\right]\right\} \\
\leq & \varphi\left(d_{\infty}\left(\mu_{j-1}, \mu_{*}\right)\right) \max \left\{d_{\infty}\left(\mu_{j-1}, \mu_{*}\right), \rho_{\infty}\left(\mu_{j-1}, F_{j}\left(\mu_{j-1}\right)\right),\right. \\
& d_{\infty}\left(\mu_{*}, \mu_{j}\right)+d_{\infty}\left(F_{j}\left(\mu_{j-1}\right), F_{i}\left(\mu_{*}\right)\right), 1 / 2\left[d_{\infty}\left(\mu_{*}, \mu_{j}\right)\right. \\
& \left.\left.+\rho_{\infty}\left(\mu_{j}, F_{j}\left(\mu_{j-1}\right)\right)+d_{\infty}\left(\mu_{j-1}, \mu_{*}\right)+\rho_{\infty}\left(\mu_{*}, F_{i}\left(\mu_{*}\right)\right)\right]\right\}
\end{aligned}
$$




$$
\begin{aligned}
\leq & \varphi\left(d_{\infty}\left(\mu_{j-1}, \mu_{*}\right)\right) \max \left\{d_{\infty}\left(\mu_{j-1}, \mu_{*}\right), \rho_{\infty}\left(\mu_{j-1}, F_{j}\left(\mu_{j-1}\right)\right),\right. \\
& d_{\infty}\left(\mu_{j}, \mu_{*}\right)+d_{\infty}\left(F_{j}\left(\mu_{j-1}\right), F_{i}\left(\mu_{*}\right)\right), 1 / 2\left[d_{\infty}\left(\mu_{j}, \mu_{*}\right)\right. \\
& \left.\left.+d_{\infty}\left(\mu_{j-1}, \mu_{*}\right)+\rho_{\infty}\left(\mu_{*}, F_{i}\left(\mu_{*}\right)\right)\right]\right\} .
\end{aligned}
$$

We now consider the following two possible cases:

Case 1. If

$$
\begin{aligned}
d_{\infty}\left(\mu_{j}, \mu_{*}\right)+d_{\infty}\left(F_{j}\left(\mu_{j-1}\right), F_{i}\left(\mu_{*}\right)\right) \\
=\max \left\{d_{\infty}\left(\mu_{j-1}, \mu_{*}\right), \rho_{\infty}\left(\mu_{j-1}, F_{j}\left(\mu_{j-1}\right)\right), d_{\infty}\left(\mu_{j}, \mu_{*}\right)\right. \\
\left.\quad+d_{\infty}\left(F_{j}\left(\mu_{j-1}\right), F_{i}\left(\mu_{*}\right)\right), 1 / 2\left[d_{\infty}\left(\mu_{j}, \mu_{*}\right)+d_{\infty}\left(\mu_{j-1}, \mu_{*}\right)+\rho_{\infty}\left(\mu_{*}, F_{i}\left(\mu_{*}\right)\right)\right]\right\},
\end{aligned}
$$

then, by inequality (3.8), we have

$$
d_{\infty}\left(F_{j}\left(\mu_{j-1}\right), F_{i}\left(\mu_{*}\right)\right) \leq \varphi\left(d_{\infty}\left(\mu_{j-1}, \mu_{*}\right)\right)\left[d_{\infty}\left(\mu_{j}, \mu_{*}\right)+d_{\infty}\left(F_{j}\left(\mu_{j-1}\right), F_{i}\left(\mu_{*}\right)\right] .\right.
$$

This is equivalent to

$$
d_{\infty}\left(F_{j}\left(\mu_{j-1}\right), F_{i}\left(\mu_{*}\right)\right) \leq \frac{\varphi\left(d_{\infty}\left(\mu_{j-1}, \mu_{*}\right)\right) d_{\infty}\left(\mu_{j}, \mu_{*}\right)}{1-\varphi\left(d_{\infty}\left(\mu_{j-1}, \mu_{*}\right)\right)} .
$$

Using, the previous argument together with inequality (3.7), we have

$$
\begin{aligned}
\rho_{\infty}\left(\mu_{*}, F_{i}\left(\mu_{*}\right)\right) & \leq d_{\infty}\left(\mu_{*}, \mu_{j}\right)+\frac{\varphi\left(d_{\infty}\left(\mu_{j-1}, \mu_{*}\right)\right) d_{\infty}\left(\mu_{j}, \mu_{*}\right)}{1-\varphi\left(d_{\infty}\left(\mu_{j-1}, \mu_{*}\right)\right)} \\
& =\frac{d_{\infty}\left(\mu_{*}, \mu_{j}\right)}{1-\varphi\left(d_{\infty}\left(\mu_{j-1}, \mu_{*}\right)\right)} .
\end{aligned}
$$

Note that, since $d_{\infty}\left(\mu_{j}, \mu_{*}\right) \rightarrow 0$ as $j \rightarrow \infty$, without loss of generality (passing to a subsequence if necessary), we may assume that $\left\{d_{\infty}\left(\mu_{j}, \mu_{*}\right)\right\}_{j=1}^{\infty}$ is a nonincreasing sequence. Subsequently, by Theorem 2.4, we have

$$
0 \leq \sup _{j \in \mathbb{N}} \varphi\left(d_{\infty}\left(\mu_{j}, \mu_{*}\right)\right)<1
$$

Subsequently, by inequality (3.9), we obtain

$$
\begin{aligned}
\rho_{\infty}\left(\mu_{*}, F_{i}\left(\mu_{*}\right)\right) & \leq \frac{d_{\infty}\left(\mu_{j}, \mu_{*}\right)}{1-\varphi\left(d_{\infty}\left(\mu_{j-1}, \mu_{*}\right)\right)} \\
& \leq \frac{d_{\infty}\left(\mu_{j}, \mu_{*}\right)}{1-\sup _{j \in \mathbb{N}} \varphi\left(d_{\infty}\left(\mu_{j-1}, \mu_{*}\right)\right)} .
\end{aligned}
$$

Letting $j \rightarrow \infty$ in inequality (3.10), we have

$$
\rho_{\infty}\left(\mu_{*}, F_{i}\left(\mu_{*}\right)\right) \leq 0
$$

This implies, by Lemma 2.2(i), that $\mu_{*} \subseteq F_{i}\left(\mu_{*}\right)$. 
Case 2. Assume

$$
\begin{aligned}
d_{\infty}\left(\mu_{j}, \mu_{*}\right)+d_{\infty}\left(F_{j}\left(\mu_{j-1}\right), F_{i}\left(\mu_{*}\right)\right) \\
\quad \neq \max \left\{d_{\infty}\left(\mu_{j-1}, \mu_{*}\right), \rho_{\infty}\left(\mu_{j-1}, F_{j}\left(\mu_{j-1}\right)\right), d_{\infty}\left(\mu_{j}, \mu_{*}\right)\right. \\
\quad+d_{\infty}\left(F_{j}\left(\mu_{j-1}\right), F_{i}\left(\mu_{*}\right)\right), 1 / 2\left[d_{\infty}\left(\mu_{j}, \mu_{*}\right)+d_{\infty}\left(\mu_{j-1}, \mu_{*}\right)\right. \\
\left.\left.\quad+\rho_{\infty}\left(\mu_{*}, F_{i}\left(\mu_{*}\right)\right)\right]\right\} .
\end{aligned}
$$

In this situation, by inequality (3.8), we have

$$
\begin{aligned}
d_{\infty} & \left(F_{j}\left(\mu_{j-1}\right), F_{i}\left(\mu_{*}\right)\right) \\
\leq & \varphi\left(d_{\infty}\left(\mu_{j-1}, \mu_{*}\right)\right) \max \left\{d_{\infty}\left(\mu_{j-1}, \mu_{*}\right), \rho_{\infty}\left(\mu_{j-1}, F_{j}\left(\mu_{j-1}\right)\right),\right. \\
& \left.1 / 2\left[d_{\infty}\left(\mu_{j}, \mu_{*}\right)+d_{\infty}\left(\mu_{j-1}, \mu_{*}\right)+\rho_{\infty}\left(\mu_{*}, F_{i}\left(\mu_{*}\right)\right)\right]\right\} \\
\leq & \varphi\left(d_{\infty}\left(\mu_{j-1}, \mu_{*}\right)\right) \max \left\{d_{\infty}\left(\mu_{j-1}, \mu_{*}\right), d_{\infty}\left(\mu_{j-1}, \mu_{j}\right), 1 / 2\left[d_{\infty}\left(\mu_{j}, \mu_{*}\right)\right.\right. \\
& \left.\left.+d_{\infty}\left(\mu_{j-1}, \mu_{*}\right)+\rho_{\infty}\left(\mu_{*}, F_{i}\left(\mu_{*}\right)\right)\right]\right\} .
\end{aligned}
$$

So, it follows by inequality (3.7) that

$$
\begin{aligned}
\rho_{\infty}\left(\mu_{*}, F_{i}\left(\mu_{*}\right)\right) \leq & d_{\infty}\left(\mu_{*}, \mu_{j}\right)+\varphi\left(d_{\infty}\left(\mu_{j-1}, \mu_{*}\right)\right) \max \left\{d_{\infty}\left(\mu_{j-1}, \mu_{*}\right), d_{\infty}\left(\mu_{j-1}, \mu_{j}\right),\right. \\
& \left.1 / 2\left[d_{\infty}\left(\mu_{j}, \mu_{*}\right)+d_{\infty}\left(\mu_{j-1}, \mu_{*}\right)+\rho_{\infty}\left(\mu_{*}, F_{i}\left(\mu_{*}\right)\right)\right]\right\} \\
\leq & d_{\infty}\left(\mu_{*}, \mu_{j}\right)+\sup _{j \in \mathbb{N}} \varphi\left(d_{\infty}\left(\mu_{j-1}, \mu_{*}\right)\right) \max \left\{d_{\infty}\left(\mu_{j-1}, \mu_{*}\right), d_{\infty}\left(\mu_{j-1}, \mu_{j}\right),\right. \\
& \left.1 / 2\left[d_{\infty}\left(\mu_{j}, \mu_{*}\right)+d_{\infty}\left(\mu_{j-1}, \mu_{*}\right)+\rho_{\infty}\left(\mu_{*}, F_{i}\left(\mu_{*}\right)\right)\right]\right\} \\
< & d_{\infty}\left(\mu_{*}, \mu_{j}\right)+\max \left\{d_{\infty}\left(\mu_{j-1}, \mu_{*}\right), d_{\infty}\left(\mu_{j-1}, \mu_{j}\right),\right. \\
& \left.1 / 2\left[d_{\infty}\left(\mu_{j}, \mu_{*}\right)+d_{\infty}\left(\mu_{j-1}, \mu_{*}\right)+\rho_{\infty}\left(\mu_{*}, F_{i}\left(\mu_{*}\right)\right)\right]\right\} .
\end{aligned}
$$

Letting $j \rightarrow \infty$ in inequality (3.11), we obtain

$$
\rho_{\infty}\left(\mu_{*}, F_{i}\left(\mu_{*}\right)\right) \leq \frac{\rho_{\infty}\left(\mu_{*}, F_{i}\left(\mu_{*}\right)\right)}{2}
$$

which implies that $\rho_{\infty}\left(\mu_{*}, F_{i}\left(\mu_{*}\right)\right)=0$. Again, by Lemma 2.2(i), it follows that $\mu_{*} \subseteq F_{i}\left(\mu_{*}\right)$. Hence, by Cases 1 and 2, the proof is completed.

Remark 3.2 Theorem 3.1 recovers Theorem 1.1 as a special case. Meanwhile, it improves Theorem 1.2 since we are considering a larger class of metric space settings.

Using Theorem 3.1, we also obtain the following result.

Corollary 3.3 Let $(X, d)$ be a complete metric space and let $\left\{F_{i}\right\}_{i=1}^{\infty}$ be a sequence of fuzzy self-mappings of $\mathcal{C B}(X)$. If there exist nonnegative constants $a, b, c, a+2 b+2 c<1$, such 
that for each $\mu_{1}, \mu_{2} \in \mathcal{C B}(X)$, and for arbitrary positive integers $i$ and $j, i \neq j$,

$$
\begin{aligned}
d_{\infty}\left(F_{i}\left(\mu_{1}\right), F_{j}\left(\mu_{2}\right)\right) \leq & a d_{\infty}\left(\mu_{1}, \mu_{2}\right)+b\left\{\rho_{\infty}\left(\mu_{1}, F_{i}\left(\mu_{1}\right)\right)+\rho_{\infty}\left(\mu_{2}, F_{i}\left(\mu_{2}\right)\right)\right\} \\
& +c\left\{\rho_{\infty}\left(\mu_{1}, F_{i}\left(\mu_{2}\right)\right)+\rho_{\infty}\left(\mu_{2}, F_{i}\left(\mu_{1}\right)\right)\right\}
\end{aligned}
$$

then there exists a $\mu_{*} \in \mathcal{C B}(X)$ such that $\mu_{*} \subseteq F_{i}\left(\mu_{*}\right)$ for all $i \in \mathbb{N}$.

Proof Let us define an $\mathcal{R}$ function $\varphi:[0, \infty) \rightarrow[0,1)$ by

$$
\varphi(t)=a+2 b+2 c \text { for all } t \in[0, \infty)
$$

Then one can derive that the relation (3.12) is transformed to (3.1), and so the required result follows immediately from Theorem 3.1.

Next, motivated by the idea of Berinde [18], we now present another fuzzy fixed point theorem.

Theorem 3.4 Let $(X, d)$ be a complete metric space and let $\left\{F_{i}\right\}_{i=1}^{\infty}$ be a sequence of fuzzy self-mappings of $\mathcal{C B}(X)$. If there exist an $\mathcal{R}$-function $\varphi:[0, \infty) \rightarrow[0,1)$ and $L \geq 0$ such that for each $\mu_{1}, \mu_{2} \in \mathcal{C B}(X)$, and for arbitrary positive integers $i$ and $j, i \neq j$,

$$
\begin{aligned}
d_{\infty}\left(F_{i}\left(\mu_{1}\right), F_{j}\left(\mu_{2}\right)\right) \leq & \varphi\left(d_{\infty}\left(\mu_{1}, \mu_{2}\right)\right) \frac{\left[\rho_{\infty}\left(\mu_{1}, F_{i}\left(\mu_{1}\right)\right)+\rho_{\infty}\left(\mu_{2}, F_{j}\left(\mu_{2}\right)\right)\right]}{2} \\
& +L \rho_{\infty}\left(\mu_{2}, F_{i}\left(\mu_{1}\right)\right)
\end{aligned}
$$

then there exists a $\mu_{*} \in \mathcal{C B}(X)$ such that $\mu_{*} \subseteq F_{i}\left(\mu_{*}\right)$ for all $i \in \mathbb{N}$.

Proof Let us construct and consider again a function $k$ and the sequences which we have defined in (3.2). Recall that, by our constructive method, we also know that

$$
\rho_{\infty}\left(\mu_{n-1}, F_{n}\left(\mu_{n-1}\right)\right) \leq d_{\infty}\left(\mu_{n-1}, \mu_{n}\right)
$$

for each $n \in \mathbb{N}$. Using this together with (3.13), we derive

$$
\begin{aligned}
d_{\infty}\left(\mu_{n+1}, \mu_{n+2}\right) \leq & \beta_{n} d_{\infty}\left(F_{n+1}\left(\mu_{n}\right), F_{n+2}\left(\mu_{n+1}\right)\right) \\
\leq & \beta_{n} \varphi\left(d_{\infty}\left(\mu_{n}, \mu_{n+1}\right)\right) \frac{\left[\rho_{\infty}\left(\mu_{n}, F_{n+1}\left(\mu_{n}\right)\right)+\rho_{\infty}\left(\mu_{n+1}, F_{n+2}\left(\mu_{n+1}\right)\right)\right]}{2} \\
& +\beta_{n} L \rho_{\infty}\left(\mu_{n+1}, F_{n+1}\left(\mu_{n}\right)\right) \\
\leq & \beta_{n} \varphi\left(d_{\infty}\left(\mu_{n}, \mu_{n+1}\right)\right) \frac{\left[d_{\infty}\left(\mu_{n}, \mu_{n+1}\right)+d_{\infty}\left(\mu_{n+1}, \mu_{n+2}\right)\right]}{2} \\
< & \beta_{n} k\left(d_{\infty}\left(\mu_{n}, \mu_{n+1}\right)\right) \frac{\left[d_{\infty}\left(\mu_{n}, \mu_{n+1}\right)+d_{\infty}\left(\mu_{n+1}, \mu_{n+2}\right)\right]}{2},
\end{aligned}
$$

for each $n \in \mathbb{N}$. This is equivalent to

$$
d_{\infty}\left(\mu_{n+1}, \mu_{n+2}\right)<\frac{\beta_{n} k\left(d_{\infty}\left(\mu_{n}, \mu_{n+1}\right)\right)}{2-\beta_{n} k\left(d_{\infty}\left(\mu_{n}, \mu_{n+1}\right)\right)} d_{\infty}\left(\mu_{n}, \mu_{n+1}\right),
$$


for each $n \in \mathbb{N}$. Observe that, since $\beta_{n} k\left(d_{\infty}\left(\mu_{n}, \mu_{n+1}\right)\right) \in(0,1)$, we also have

$$
\frac{\beta_{n} k\left(d_{\infty}\left(\mu_{n}, \mu_{n+1}\right)\right)}{2-\beta_{n} k\left(d_{\infty}\left(\mu_{n}, \mu_{n+1}\right)\right)} \in(0,1)
$$

for all $n \in \mathbb{N}$. Thus, by (3.14), we see that $\left\{d_{\infty}\left(\mu_{n}, \mu_{n+1}\right)\right\}$ is a strictly decreasing sequence in $[0, \infty)$. Since $\varphi$ is an $\mathcal{R}$-function, by applying Theorem 2.4 , we have

$$
0 \leq \sup _{n \in \mathbb{N}} \varphi\left(d_{\infty}\left(\mu_{n}, \mu_{n+1}\right)\right)<1 .
$$

Next, as we have done in order to prove Theorem 3.1, we write

$$
\lambda:=\sup _{n \in \mathbb{N}} \beta_{n} k\left(d_{\infty}\left(\mu_{n}, \mu_{n+1}\right)\right)<1 .
$$

Let us take $c:=\frac{\lambda}{2-\lambda}$. It follows that $c \in(0,1)$ and, by (3.14), we also have

$$
\begin{aligned}
d_{\infty}\left(\mu_{n+1}, \mu_{n+2}\right) & <\frac{\beta_{n} k\left(d_{\infty}\left(\mu_{n}, \mu_{n+1}\right)\right)}{2-\beta_{n} k\left(d_{\infty}\left(\mu_{n}, \mu_{n+1}\right)\right)} d_{\infty}\left(\mu_{n}, \mu_{n+1}\right) \\
& \leq c d_{\infty}\left(\mu_{n}, \mu_{n+1}\right) .
\end{aligned}
$$

Now, again by reasoning along the lines of proving Theorem 3.1, we can show that there is a $\mu^{*} \in \mathcal{C B}(X)$ which is the limit point of the considered sequence $\left\{\mu_{n}\right\}$, under the supremum metric $d_{\infty}$, and it satisfies

$$
0 \leq \sup _{j \in \mathbb{N}} \varphi\left(d_{\infty}\left(\mu_{j}, \mu_{*}\right)\right)<1
$$

Finally, we will show that $\mu_{*} \subseteq F_{i}\left(\mu_{*}\right)$ for all $i \in \mathbb{N}$. Let $i \in \mathbb{N}$ be arbitrary. Note again that

$$
\begin{aligned}
\rho_{\infty}\left(\mu_{*}, F_{i}\left(\mu_{*}\right)\right) & \leq d_{\infty}\left(\mu_{*}, \mu_{j}\right)+\rho_{\infty}\left(\mu_{j}, F_{i}\left(\mu_{*}\right)\right) \\
& \leq d_{\infty}\left(\mu_{*}, \mu_{j}\right)+d_{\infty}\left(F_{j}\left(\mu_{j-1}\right), F_{i}\left(\mu_{*}\right)\right),
\end{aligned}
$$

for each $j \in \mathbb{N}$ such that $j \neq i$. Consider

$$
\begin{aligned}
d_{\infty} & \left(F_{j}\left(\mu_{j-1}\right), F_{i}\left(\mu_{*}\right)\right) \\
\leq & \varphi\left(d_{\infty}\left(\mu_{j-1}, \mu_{*}\right)\right) \frac{\left[\rho_{\infty}\left(\mu_{j-1}, F_{j}\left(\mu_{j-1}\right)\right)+\rho_{\infty}\left(\mu_{*}, F_{i}\left(\mu_{*}\right)\right)\right]}{2}+L \rho_{\infty}\left(\mu_{*}, F_{j}\left(\mu_{j-1}\right)\right) \\
\leq & \varphi\left(d_{\infty}\left(\mu_{j-1}, \mu_{*}\right)\right) \frac{d_{\infty}\left(\mu_{j-1}, \mu_{j}\right)}{2}+\varphi\left(d_{\infty}\left(\mu_{j-1}, \mu_{*}\right)\right) \frac{\rho_{\infty}\left(\mu_{*}, F_{i}\left(\mu_{*}\right)\right)}{2} \\
& +L\left(d_{\infty}\left(\mu_{*}, \mu_{j}\right)+\rho_{\infty}\left(\mu_{j}, F_{j}\left(\mu_{j-1}\right)\right)\right) \\
\leq & \varphi\left(d_{\infty}\left(\mu_{j-1}, \mu_{*}\right)\right) \frac{d_{\infty}\left(\mu_{j-1}, \mu_{j}\right)}{2}+\varphi\left(d_{\infty}\left(\mu_{j-1}, \mu_{*}\right)\right) \frac{\rho_{\infty}\left(\mu_{*}, F_{i}\left(\mu_{*}\right)\right)}{2} \\
& +L d_{\infty}\left(\mu_{*}, \mu_{j}\right)+L \rho_{\infty}\left(\mu_{j}, F_{j}\left(\mu_{j-1}\right)\right),
\end{aligned}
$$


where $j \in \mathbb{N}$ with $j \neq i$. Subsequently, from (3.17) together with (3.16), we obtain

$$
\begin{aligned}
\rho_{\infty}\left(\mu_{*}, F_{i}\left(\mu_{*}\right)\right) \leq & d_{\infty}\left(\mu_{*}, \mu_{j}\right)+\varphi\left(d_{\infty}\left(\mu_{j-1}, \mu_{*}\right)\right) \frac{d_{\infty}\left(\mu_{j-1}, \mu_{j}\right)}{2} \\
& +\varphi\left(d_{\infty}\left(\mu_{j-1}, \mu_{*}\right)\right) \frac{\rho_{\infty}\left(\mu_{*}, F_{i}\left(\mu_{*}\right)\right)}{2} \\
& +L d_{\infty}\left(\mu_{*}, \mu_{j}\right)+L \rho_{\infty}\left(\mu_{j}, F_{j}\left(\mu_{j-1}\right)\right) \\
\leq & (1+L) d_{\infty}\left(\mu_{*}, \mu_{j}\right)+\sup _{j \in \mathbb{N}} \varphi\left(d_{\infty}\left(\mu_{j}, \mu_{*}\right)\right) \frac{d_{\infty}\left(\mu_{j-1}, \mu_{j}\right)}{2} \\
& +\sup _{j \in \mathbb{N}} \varphi\left(d_{\infty}\left(\mu_{j-1}, \mu_{*}\right)\right) \frac{\rho_{\infty}\left(\mu_{*}, F_{i}\left(\mu_{*}\right)\right)}{2} \\
< & (1+L) d_{\infty}\left(\mu_{*}, \mu_{j}\right)+\frac{d_{\infty}\left(\mu_{j-1}, \mu_{j}\right)}{2}+\frac{\rho_{\infty}\left(\mu_{*}, F_{i}\left(\mu_{*}\right)\right)}{2} .
\end{aligned}
$$

By letting $j \rightarrow \infty$ on the right of inequality (3.18), we have

$$
\rho_{\infty}\left(\mu_{*}, F_{i}\left(\mu_{*}\right)\right) \leq \frac{\rho_{\infty}\left(\mu_{*}, F_{i}\left(\mu_{*}\right)\right)}{2},
$$

which implies that $\rho_{\infty}\left(\mu_{*}, F_{i}\left(\mu_{*}\right)\right)=0$. Then, by Lemma 2.2(i), we have $\mu_{*} \subseteq F_{i}\left(\mu_{*}\right)$. Since $i \in \mathbb{N}$ is arbitrary, we complete the proof.

The following result can be deduced from Theorem 3.4.

Corollary 3.5 Let $(X, d)$ be a complete metric space and let $\left\{F_{i}\right\}_{i=1}^{\infty}$ be a sequence of fuzzy self-mappings of $\mathcal{C B}(X)$. If there exist an $\mathcal{R}$-function $\varphi:[0, \infty) \rightarrow[0,1)$ and $L \geq 0$ such that for each $\mu_{1}, \mu_{2} \in \mathcal{C B}(X)$, and for arbitrary positive integers $i$ and $j, i \neq j$,

$$
\begin{aligned}
d_{\infty}\left(F_{i}\left(\mu_{1}\right), F_{j}\left(\mu_{2}\right)\right) \leq & \varphi\left(d_{\infty}\left(\mu_{1}, \mu_{2}\right)\right)\left[\rho_{\infty}\left(\mu_{1}, F_{i}\left(\mu_{1}\right)\right) \rho_{\infty}\left(\mu_{2}, F_{j}\left(\mu_{2}\right)\right)\right]^{1 / 2} \\
& +L \rho_{\infty}\left(\mu_{2}, F_{i}\left(\mu_{1}\right)\right)
\end{aligned}
$$

then there exists a $\mu_{*} \in \mathcal{C B}(X)$ such that $\mu_{*} \subseteq F_{i}\left(\mu_{*}\right)$ for all $i \in \mathbb{N}$.

Proof Since for each $a, b \in \mathbb{R}^{+}$, we have $\sqrt{a b} \leq \frac{a+b}{2}$, the required result follows immediately from our Theorem 3.4.

Remark 3.6 Corollary 3.5 recovers a result which has presented in [12], when $L=0$.

\section{Conclusions}

In this paper we have presented fuzzy fixed point theorems on the space of fuzzy sets under a kind of supremum metric setting. We would like to point out that this kind of space is very general and interesting. Moreover, as one can observe, a fixed point in this situation is, in fact, a fixed (fuzzy) set. This means that our presented results are very general and we recover many existing results on fixed point theory as regards both single-valued and set-valued mappings. 
Authors' contributions

All authors contributed equally to the writing of this paper. All authors read and approved the final manuscript.

\section{Author details}

'Department of Mathematics, Faculty of Science, Chiang Mai University, Chiang Mai, 50200, Thailand. ${ }^{2}$ Department of Mathematics, Faculty of Science, Naresuan University, Phitsanulok, 65000, Thailand. ${ }^{3}$ Research Center for Academic Excellence in Mathematics, Faculty of Science, Naresuan University, Phitsanulok, 65000, Thailand.

\section{Acknowledgements}

This paper is partially supported by the Thailand Research Fund under the project RTA5780007. N Petrot is partially supported by Naresuan University Project R2558C098. W Saksirikun is supported by the Thailand Research Fund through the Royal Golden Jubilee Ph.D. Program (Grant No. PHD/0248/2553).

Received: 15 May 2015 Accepted: 6 September 2015 Published online: 17 September 2015

\section{References}

1. Zadeh, LA: Fuzzy sets. Inf. Control 8, 338-353 (1965)

2. Heilpern, S: Fuzzy mappings and fixed point theorem. J. Math. Anal. Appl. 83, 566-569 (1981)

3. Estruch, VD, Vidal, A: A note on fixed fuzzy points for fuzzy mappings. Rend. Ist. Mat. Univ. Trieste 32, $39-45$ (2001)

4. Sedghi, S, Shobe, N, Altun, I: A fixed fuzzy point for fuzzy mappings in complete metric spaces. Math. Commun. 13 289-294 (2008)

5. Anastassiou, GA: Multivariate fuzzy perturbed neural network operators approximation. J. Nonlinear Sci. Appl. 7(6), 383-406 (2014)

6. Azama, A, Beg, l: Common fuzzy fixed points for fuzzy mappings. Fixed Point Theory Appl. 2013, 14 (2013)

7. Bose, RK, Sahani, D: Fuzzy mappings and fixed point theorems. Fuzzy Sets Syst. 21, 53-58 (1987)

8. Butnariu, D: Fixed point for fuzzy mapping. Fuzzy Sets Syst. 7, 191-207 (1982)

9. Kaur, S, Kaur, J: Integrability and $L^{1}$-convergence of fuzzy trigonometric series with special fuzzy coefficients. J. Nonlinear Sci. Appl. 8(1), 23-39 (2015)

10. Lee, BS, Cho, SJ: A fixed point theorem for contractive type fuzzy mappings. Fuzzy Sets Syst. 61, 309-312 (1994)

11. Moreno, JM, Roldan, A, Roldan, C, Cho, YJ: Multi-dimensional coincidence point theorems for weakly compatible mappings with the CLRg-property in (fuzzy) metric spaces. Fixed Point Theory Appl. 2015, 53 (2015)

12. Qiu, D, Shu, L: Supremum metric on the space of fuzzy sets and common fixed point theorems for fuzzy mappings. Inf. Sci. 178, 3595-3604 (2008)

13. Rashwan, RA, Ahmad, MA: Common fixed point theorems for fuzzy mappings. Arch. Math. 38, $219-226$ (2002)

14. Turkoglu, D, Rhoades, BE: A fixed fuzzy point for fuzzy mapping in complete metric spaces. Math. Commun. 10, 115-121 (2005)

15. Qiu, D, Shu, L, Guan, J: Common fixed point theorems for fuzzy mappings under $\Phi$-contraction condition. Chaos Solitons Fractals 41, 360-367 (2009)

16. Diamond, P, Kloeden, P: Metric Spaces of Fuzzy Sets Theory and Applications. World Scientific, Singapore (1994)

17. Du, W-S: On coincidence point and fixed point theorems for nonlinear multivalued maps. Topol. Appl. 159, 49-56 (2012)

18. Berinde, V: Approximating fixed points of weak contractions using Picard iteration. Nonlinear Anal. Forum 9(1), 43-53 (2004)

\section{Submit your manuscript to a SpringerOpen ${ }^{\odot}$ journal and benefit from:}

- Convenient online submission

Rigorous peer review

- Immediate publication on acceptance

- Open access: articles freely available online

- High visibility within the field

- Retaining the copyright to your article 